\title{
Advances in psychiatric treatment in primary care
}

\author{
Christopher Dowrick
}

Following ground-breaking work by Shepherd et al (1966) and, more recently, Goldberg \& Huxley (1992), primary care is now recognised as the arena in which most contact occurs between the National Health Service (NHS) and people with mental health problems. General practitioners (GPs) remain the first, and in many cases the only, health professionals involved in the management of a whole range of conditions, from common anxiety and depressive disorders to severe and enduring mental illnesses.

Recent contributions to Advances in Psychiatric Treatment have discussed methods of working between psychiatrists and primary care professionals and considered the psychiatric management of children and adolescents in primary care settings. This paper will focus on three topics of particular importance in primary care with regard to the psychiatric problems experienced by adults: depression and related disorders; severe and enduring mental illness; and patients with unexplained physical symptoms.

\section{Depression and related disorders}

\section{Systematic approaches to disease management}

Endeavours to improve the overall ability of GPs and primary care teams to detect and manage depression can be categorised into two broad themes: training programmes for health professionals and improvements in the organisation of care pathways.
Training programmes

There has been considerable interest in the potential benefits of providing educational and training programmes for GPs. This was stimulated initially by a study conducted on the Swedish island of Gotland, with a population of some 60000 (Rutz et $a l, 1989)$. A series of didactic seminars for 18 primary care physicians appeared to lead to a decrease in sickness absence from work, hospital referrals and suicide rates, and to an increase in the rate of antidepressant prescribing. However, this study was not a controlled trial, and it was unclear what proportion of such changes reflected a real reduction in psychiatric morbidity, rather than simply an alteration in the doctor's management behaviours.

Two randomised controlled trials testing the effects of primary care training on outcomes for patients with depression have now been conducted in England. Neither has been able to replicate the positive findings of the Gotland study.

The Hampshire Depression Project assessed the effectiveness in a sample of 60 primary care practices of an educational programme based on a clinical practice guideline for depression (Thompson et al, 2000). Education was delivered to all members of the primary health care teams; the main outcome measures were recognition of depression and clinical improvement. The education was well-received, but the intervention had no apparent effect either on GPs' recognition of depressive symptoms or on the outcome of depression at either 6 weeks or 6 months.

A trial in north-west England, involving 38 GPs from Merseyside and Manchester, evaluated a 10hour depression training course that included lectures, role-play with video feedback and group discussion (Gask et al, 1998). The content of this programme is outlined in Box 1. Patients' depression status was

Christopher Dowrick is Professor of Primary Medical Care and Head of the Department of Primary Care in the University of Liverpool, and a part-time general practitioner with the Aintree Park Group Practice. His main research interests are in mental health in community and primary care settings, and in the interactions between doctors and patients. 
Box 1 The assessment and management of depression in primary care: a 10 -hour course for general practitioners (GPs) devised by Gask et al (1998)

Aims

To improve the assessment and management of depression in general practice by effective use of pharmacological, physical and social interventions that are realistic within the confines of the consultation

Training methods

The key focus of the course is on acquisition of appropriate clinical skills. Each of the five 2-hour sessions includes:

- a brief presentation/lecture on each topic

- viewing specially developed videotapes

- the opportunity for each GP to role-play consultations as both GP and patient

- discussion of the videotaped role-plays in small feedback groups

- written material to support each session

Course timetable

Week 1 Assessing depression

Week 2 Negotiating the treatment contract and drug treatment of depression

Week 3 Problem-solving therapy and social interventions

Week 4 The question of suicide

Week 5 Cognitive and behavioural skills

the primary outcome measure, with secondary measures of depressive symptoms, subjective function, patient satisfaction and patterns of health care utilisation. No overall differences were revealed in any of the outcome measures between intervention and control groups, at 3 or 12 months. The study found that the Manchester GPs had less prior training and experience in mental health than those from Merseyside, and there was a consistent pattern for the training effect to be greater in the former than the latter (further details available from the author upon request). This suggests that ways must be found of targeting training at those doctors who are most in need of it.

In terms of the specific and important problem of suicide prevention, educational interventions may yet prove to be of benefit. In a pilot study for the STORM project (Appelby et al, 2000), an educational programme for 'frontline' staff in primary care, accident and emergency units and community mental health teams was associated with a subsequent $14 \%$ drop in suicide in following year in the health district where the study took place, at a time when the national suicide rate increased by almost $4 \%$.
However, this study needs to be replicated on a wider scale, and under controlled conditions, before such benefits can be confidently claimed.

Organising care pathways

A series of studies undertaken in primary care clinics in Washington, DC, to determine the efficacy and cost-effectiveness of formal systematic approaches to the management of depression in primary care has yielded some promising results.

Katon et al (1995) set up a multifaceted treatment programme, which included giving booklets and videotapes to patients with depression, involving GPs in training sessions and case conferences, and introducing a structured series of extended patient visits with GPs and psychiatrists. This programme was effective in treating cases of major depression, but it involved extra treatment costs of between $£ 185$ and $£ 375$ per patient, which raised resource concerns within the US insurance-based health system.

More recently, Simon et al (2000) reported significant benefits, at lower cost, from a telephone care management system. Patients starting antidepressant treatment were randomised in a trial to receive either usual GP care, treatment feedback to GPs, or feedback to GPs plus care management. The last was delivered by a care manager with no specific training in antidepressant pharmacotherapy: it included two 10- to 15-minute telephone assessments of each patient, 8 and 16 weeks after the initial prescription, followed by sophisticated treatment recommendations for the GP, and support for the doctors in implementing these recommendations. At an incremental cost of $£ 50$ per patient, those patients in the 'feedback plus care management' arm of the trial had a higher probability of receiving at least a moderate dose of antidepressants and a 50\% improvement in depression checklist scores and a lower probability of major depression at follow-up.

Although undertaken in the USA, in the context of a health care system quite different from that found in the UK, these studies may well have interesting applications for primary care psychiatry in this country. Systematic telephone contact, with feedback and support for GPs, could be undertaken by practice nurses within a single practice, or by a small group of dedicated mental health care staff attached to a community mental health team or situated within the broader framework of a primary care group or trust.

\section{Patterns of antidepressant prescribing}

The number of prescriptions of antidepressant drugs issued to patients with a diagnosis of depression in primary care in the UK increased by $33 \%$ between 1993 and 1995, with a particularly rapid escalation 
(134\%) among the selective serotonin reuptake inhibitors (SSRIs) (Donoghue et al, 1996). It is not yet clear whether this change in the prescribing habits of GPs represents a major treatment advance, or a triumph for pharmaceutical marketing strategies.

There is substantial agreement that the major antidepressant groups, if provided in adequate doses and for long enough periods, are of broadly equal efficacy in reducing the symptoms of major depression. Much of the recent debate on the relative merits of different groups of drugs has centred on their side-effect profiles and their cost-effectiveness.

Simon et al (1996) compared fluoxetine and imipramine in primary care settings in Seattle. They found fewer adverse effect reports and lower dropout rates with fluoxetine, but no overall differences in clinical or quality of life outcomes. They reported similar total health care costs, with higher fluoxetine drug costs balanced by fewer out-patient and inpatient contacts with medical and mental health services. The North of England Evidence-Based Guideline Development Project (Eccles et al, 1999) undertook a detailed cost-benefit analysis of the direct health care costs of the major antidepressant groups, including drug costs, toxicity admissions and notional lives saved. Their meta-analysis of existing studies concluded that tricyclic antidepressants should be used as routine first-line treatment for depression in primary care, with lofepramine as a more cost-effective option than an SSRI if toxic effects of the first-generation tricyclics are perceived to be a problem. It may be relevant to note that the first of these studies was funded by Lilly Research Laboratories and the second by the Prescribing Research Initiative of the UK Department of Health.

General practitioners, in no small measure prompted by their patients, are becoming increasingly aware of the potential of complementary drug therapies. In particular, St John's wort (Hypericum perforatum), a herbal treatment commonly prescribed in Germany for mild and moderate depression, has become such a popular over-the-counter treatment for depression that it is attracting the attention of the NHS Committee for the Safety of Medicines. Recent research evidence from a study based in primary care in four German states (Philipp et al, 1999) indicates that $1050 \mathrm{mg} /$ day hypericum extract - which is thought to be the main active ingredient of St John's wort - is at least as effective as $100 \mathrm{mg} /$ day imipramine in the treatment of moderate depression, in terms of both reduction in depressive symptoms and improved quality of life scores up to 2 months after beginning treatment. Importantly, the rate of reported adverse effects with hypericum (22\%) was significantly lower than the rate for imipramine (46\%), which suggests that the herbal treatment may have an overall therapeutic advantage. A large randomised controlled trial of hypericin, sertraline and placebo is currently underway in the USA, funded by the National Institute for Mental Health. It may be prudent to await the results of this trial before proposing that St John's wort be included as a first-line treatment for depression in primary care.

\section{Psychosocial interventions}

It has been difficult, until recently, to evaluate the role of psychological interventions in managing depression and related disorders in primary care. In part this has been due to methodological weakness in study design and to the inherent difficulty of defining treatment content and appropriate outcome measures (Corney, 1992). The preference for such therapies expressed by both patients and physicians can also adversely affect recruitment (Fairhurst \& Dowrick, 1996). Several well-designed trials have been published that provide credible evidence of clinical and cost-effectiveness, at least in the short-term, for specific interventions such as cognitive-behavioural (Appelby et al, 1997; Scott et al, 1997) and interpersonal (Shea et al, 1992) therapies. However, these interventions are somewhat complex and involve relatively lengthy training periods, and it is not yet clear how readily applicable they may be in primary care settings.

There has been considerable recent research interest in the UK in the potential of problem-solving treatment as a primary care intervention for depression. This treatment is based on the observation that emotional symptoms are generally induced by problems of living. It has been developed as a specific, collaborative process, with three main steps: first, patients' symptoms are linked with their problems; second, the problems are defined and clarified; and third, an attempt is made to solve the problems in a structured way (Box 2). By starting to tackle their problems, patients can begin to reassert control over their lives, and it is proposed that it is this regaining of control that lifts mood. The process usually involves six sessions with a therapist, with a total contact time of less than 4 hours.

The skills needed to deliver problem-solving treatment can be easily and quickly taught to a range of health professionals, including GPs and nurses. It has been shown to be useful in primary care for patients experiencing anxiety and minor emotional disorders (Catalan et al, 1991). In two trials conducted by Mynors-Wallis et al (1995, 2000), problem-solving treatment was found to be as effective as antidepressant medication in the treatment of major depression, when provided either by experienced GPs or by primary care nurses. In the Outcomes of Depression International Network (ODIN) project (Dowrick et al, 1998), a randomised controlled trial was undertaken 
Box 2 Problem-solving handout for patients, devised by Mynors-Wallis et al (1995)

Depression is very common. It is often induced by problems of living. Depressed people can learn ways of dealing with these problems. Using problem-solving, they can learn to cope better and reduce their depressive symptoms.

Problem-solving is a systematic, common-sense way of sorting out problems and difficulties. If you learn how to do problem solving, you can lessen your depressive symptoms without having to take pills. In problem-solving, the therapist explains the details of the treatment and provides encouragement and support, but the ideas and plans come from you. Problem-solving will be useful to you not only now but also in the future if problems arise.

There are five important stages:

1 Write down a clear description of the main problem. What is the nature of the problem? When does the problem occur? Where does the problem occur? Try to break up complicated problems into several smaller problems and consider each separately.

2 Decide on your goals. Choose achievable and definite goals.

3 List as many alternative solutions as you can, before considering the pros and cons for each one.

4 Choose your best solution.

5 Set out clear steps to achieve the solution: specify exactly what you are going to do and when.

Problem-solving concentrates on the here and now rather than on mistakes in the past. You should focus on improving the future rather than regretting the past.

Problem-solving may not solve all your difficulties, but it can help you to start dealing with your problems. As your symptoms improve you will feel more and more in control of the problems.

comparing problem-solving treatment with a group intervention for people with depressive disorders identified through community surveys in six European countries. Problem-solving treatment appeared acceptable to subjects and led to a reduction in the duration and severity of depressive disorders and an improvement in subjective function.

\section{Severe and enduring mental illness}

During the past decade there has been considerable interest in developing effective case-management approaches for patients with severe and enduring mental illness (including schizophrenia, bipolar affective disorders and persistent or recurrent anxiety and depressive disorders), based in primary care. These have arisen as a result of the major shift in emphasis from hospital-based to communitybased care for this group of vulnerable patients, and an awareness that primary care teams have often lacked the skills (and sometimes the motivation) to provide effective care management. They may be seen as part of a broader set of intervention strategies which have the common aim of reducing the distress and disability so often resulting from these conditions. There is, in theory, a major role for primary care teams in the follow-up care of patients who have recovered from acute episodes. However, the evidence to date regarding the efficacy or otherwise of such primarycare-based strategies is inconsistent.

Nazareth et al (1996) have evaluated the feasibility, acceptability and effectiveness of a structured approach to the management of schizophrenia in general practice. They recruited all patients with non-affective psychosis in four inner-city practices. In two of the four practices, they gave GPs and practice nurses a checklist and set of outcome measures to use when reviewing these patients. They found wide variations in the proportion of patients attending for review. In the intervention practices, there were significant increases in consultation rates and

\section{Box 3 Depression: a summary}

Well-focused training of health professionals can achieve health gain for patients

Systematic primary care management has produced positive benefits in the USA

Tricyclic antidepressants still have a place in prescribing, and there is evidence of efficacy for St John's wort

Psychosocial interventions are as effective as antidepressants 
significant improvements on the Global Assessment Scale (Endicott et al, 1976) and on the 'behaviour', 'speech' and 'other syndromes' subscales of the Present State Examination (Wing et al, 1974). They concluded that health surveillance of patients with non-affective psychosis is possible in general practice.

Kendrick et al (1995) tested the teaching to GPs of structured assessments of patients with long-term mental illness (Box 4). They found that changes in treatment with neuroleptic drugs and referrals to community psychiatric nurses were significantly more frequent in the intervention group, but that few patients received the assessments after the first 6 months. They concluded that such training for GPs can increase their involvement in psychiatric care, but regular structured assessments were not feasible in routine surgeries.

They then evaluated the impact of a similar structured assessment, this time delivered by practice nurses after a 1-day training course (Burns et al, 1998). They demonstrated a high rate of consultation and clinical need in this patient group.
The practice nurses were more diligent than GPs in carrying out assessments, but there was no impact on treatment patterns or clinical outcome.

The implication of these research studies appears to be that structured assessments by GPs and nurses with this patient group may well be feasible, but further training is needed if this intervention is to be translated into health gain. They also revealed a pressing need for development of better integrated approaches between primary care and community mental health teams (Gask \& Croft, 2000), particularly within the new organisational context of primary care groups and trusts.

\section{Unexplained physical symptoms}

General practitioners have long been aware of a substantial proportion of patients who present with

Box 4 General practitioners' (GPs') structured assessment card for people with severe and enduring mental illness, devised by Kendrick et al (1995)

[front ofform]

Name

First diagnosed

Hospital admissions

Relatives/other carers

GP

Consultant

Psychologist

Pattern of relapse

Important notes

[back ofform]

Medication

Anxiety

Depression

Hallucinations

Delusions

Appearance

Behaviour

Preventive

Cardiovascular

Respiratory

Other physical

Housing

Finance

Occupation

Social life
D.O.B.

Hospital No.

Community psychiatric nurse

Social worker

Occupational therapist 
Box 5 Severe and enduring mental illness: a summary

- Primary care teams may participate in follow-up care of patients with severe and enduring mental illness

- Structured management by general practitioners and nurses is feasible, but health gain is unproven

persistent physical symptoms, unexplained by physical pathology. They receive disproportionate amounts of ineffective primary and secondary care that is often iatrogenic, by increasing dependence on medical services. In a study by Peveler et al (1997), GPs identified clinically significant physical symptoms of at least 3 months' duration resulting in social or physical disability, but without physical disease, in $19 \%$ of consecutive consultations. These patients are also recognisable in literature on frequent attenders (Dowrick et al, 2000) and are usually as disabled as patients with severe chronic disease.

Medicine has tended to explain these symptoms in terms of somatisation, the assumption being that they express underlying depression or anxiety. However, while evidence of depression or anxiety is common in patients with unexplained symptoms, it is not ubiquitous. A detailed primary care study of 228 patients with persistent unexplained symptoms (Salmon et al, 1999) and a historical study of a single family of frequent attenders (Dowrick, 1992) together suggest that such presentations can be understood as a product neither of psychopathology nor of discrete functional syndromes such as chronic fatigue. Instead, it may be that doctor-patient interactions are crucial in the aetiology of persistent unexplained symptoms.

It is typically assumed that the essence of the doctor-patient relationship is collaboration in addressing the clinical condition by appropriate information-gathering and successful treatment. However, a striking finding from interviews with patients with unexplained symptoms is that doctors are not necessarily valued or deprecated according to whether they succeed or fail to diagnose and treat patients' symptoms (Peters et al, 1998). To understand this, it is necessary to appreciate that these patients viewed symptoms as expressions of malign and menacing entities, separate from themselves. That is, symptoms were seen as having an existence outside the body, invading the body and moving around within it, changing form and evading capture. In facing this threat, patients valued doctors who provided one or both of two functions: naming the symptoms; and/or establishing an alliance against the symptoms.

In many respects, such patients consider themselves to be more expert than their doctors (Peters et $a l, 1998)$. They derive this sense of superior expertise from four main factors: their belief in the infallibility of their direct sensory experience of their symptoms, by comparison with doctors' reliance on indirect, and therefore fallible, results of medical tests; the critical evaluation of evidence and scrutiny of alternative explanations, including those provided by doctors; their perception that doctors deny the reality of their symptoms; their perception that doctors are incompetent and damaging. Further exploratory studies are needed to understand the nature of the relationships between patients and doctors in these circumstances, and particularly to understand the mechanisms by which patients seek to influence doctors towards somatic investigations, treatments or referrals.

General practitioners view dealing with medically unexplained symptoms as their second most important training need in mental health (Kerwick et al, 1997). In practical terms, there is increasing evidence that certain types of reattribution training may be helpful with this group of patients. Reattribution training is delivered to GPs in a format similar to that described above (Box 1; Gask et al, 1998). The training aims to enable doctors to recognise and manage four crucial stages in the consultation process.

Feeling understood - the GP elicits a history of the physical symptoms, explores the patient's beliefs about them and about associated psychosocial and lifestyle factors and carries out a brief focused physical examination.

Broadening the agenda - the doctor feeds back the results of the examination and any recent investigations, and explains the lack of serious underlying pathology, thus broadening the agenda beyond the presenting physical symptoms. The doctor explicitly acknowledges the reality of the patient's physical symptoms, and explores the extent of the patient's acceptance that psychosocial or lifestyle factors may be linked to them.

Making the link - the doctor relates the physical symptoms to an underlying psychosocial or lifestyle explanation, using physiological and/or temporal links that are compatible with the patient's symptom beliefs.

Negotiation over further management - there are various components to this stage, including exploring the patient's views about treatment, promoting problem-solving and coping strategies, appropriate use of muscle relaxation and/or treatment for depression, and agreeing specific plans for follow-up. 
Box 6 Unexplained physical symptoms: a summary

- These are common in primary care

- They are often associated with communication problems between doctors and patients

- Reattribution training may be helpful

Morriss et al $(1998,1999)$ have conducted a study of reattribution training $v$. treatment as usual, as taught to eight GPs who then treated 215 patients with medically unexplained symptoms. In terms of patient outcomes, they found that the training significantly improved function and reduced mental disorder among the majority of patients who did not have fixed beliefs that their symptoms had a physical cause. The change was apparent at 1 month and greatest at 3 months. Among patients who did have a fixed belief that their symptoms had a physical cause, the training significantly reduced the prevalence of major depression by $50 \%$ at 1 month. They found that reattribution training reduced the costs of health contacts outside the primary health care team by $23 \%$, with very little change in primary care costs. Overall health costs were reduced by $15 \%$ over 3 months. This reduction in health contacts with outside agencies is particularly important. The ICD-10 (World Health Organization, 1992) category 'symptoms and signs of ill-defined conditions' accounts for the greatest NHS hospital out-patient expenditure for any single category, at over $£ 200$ million per year. Many of these patients could be better and more cost-effectively managed in primary care, with an estimated saving from reattribution training of up to $£ 30$ million per year.

\section{Conclusions}

During the past decade there has been a major investment of time and effort in improving the ability of GPs and their colleagues in primary care teams to recognise and manage mental health problems more effectively. Although GP training programmes have thus far proved of limited value in improving outcomes for depression and related disorders, there are indications that they have potential benefit for suicide prevention and for patients with medically unexplained symptoms. Structured approaches to primary care management of depression are showing promise, although the evidence of their efficacy is less convincing for patients with severe and enduring mental illness. Faced with an apparently inexorable rise in antidepressant prescribing, it is timely to stress the acceptability to patients, and the effectiveness, of alternative psychological interventions.

\section{References}

Appelby, L., Warner, R., Whitton, A., et al (1997) A controlled study of fluoxetine and cognitive-behavioural counselling in the treatment of postnatal depression. British Medical Journal, 314, 932-936.

--, Morriss, R., Gask, L., et al (2000) An educational intervention for front-line health professionals in the assessment and management of suicidal patients (the STORM Project). Psychological Medicine, 30, 805-812.

Burns, T., Millar, E., Garland, C., et al (1998) Randomized controlled trial of teaching practice nurses to carry out structured assessments of patients receiving depot antipsychotic medication. British Journal of General Practice, 48,1845-1848.

Catalan, J., Gath, D., Anastasiades, P., et al (1991) Evaluation of a brief psychological treatment for emotional disorders in primary care. Psychological Medicine, 21, 1013-1018.

Corney, R. (1992) The effectiveness of counselling in general practice. International Review of Psychiatry, 4, 331-338.

Donoghue, J., Tylee, A. \& Wildgust, H. (1996) Cross sectional database analysis of antidepressant prescribing in general practice in the United Kingdom 1993-5. British Medical Journal, 313, 861-862.

Dowrick, C. (1992) Why do the O'Sheas consult so often? An exploration of complex family illness behaviour. Social Science and Medicine, 34, 491-497.

--, Casey, P., Dalgard, O., et al (1998) Outcomes of Depression International Network (ODIN). Background, methods and field trials. ODIN Group. British Journal of Psychiatry, 172, 359-363.

--, Bellon, J. A. \& Gomez, M. J. (2000) GP frequent attendance in Liverpool and Granada: the impact of depressive symptoms. British Journal of General Practice, 50, 361-365.

Eccles, M., Freemantle, N., Mason, J., et al (1999) North of England Evidence-Based Guideline Development Project: summary version of guidelines for the choice of antidepressants for depression in primary care. Family Practice, 16, 103-111.

Endicott, J., Spitzer, R. L., Fleiss, J. L., et al (1976) The global assessment scale: a procedure for measuring overall severity of psychiatric disturbance. Archives of General Psychiatry, 33, 766-771.

Fairhurst, K. \& Dowrick, C. (1996) Problems with recruitment in a randomised controlled trial of counselling in general practice: causes and implications. Journal of Health Services Research and Policy, 1, 77-80.

Gask, L., Usherwood, T., Thompson, H., et al (1998) Evaluation of a training package in the assessment and management of depression in primary care. Medical Education, 32, 190-198.

- \& Croft, J. (2000) Methods of working with primary care. Advances in Psychiatric Treatment, 6, 442-449.

Goldberg, D. \& Huxley, P. (1992) Common Mental Disorders: A Bio-Social Model. London: Routledge.

Katon, W., Von Korff, M., Lin, E., et al (1995) Collaborative management to achieve treatment guidelines. Impact on depression in primary care. Journal of the American Medical Association, 273, 1026-1031.

Kendrick, T., Burns, T. \& Freeling, P. (1995) Randomised controlled trial of teaching general practitioners to carry our structured assessments of their long term mentally ill patients. British Medical Journal, 311, 93-98.

Kerwick, S., Jones, R., Mann, A., et al (1997) Mental health care training priorities in general practice. British Journal of General Practice, 47, 225-227. 
Morriss, R., Gask, L., Ronalds, C., et al (1998) Costeffectiveness of a new treatment for somatised mental disorders taught to general practitioners. Family Practice, 15, 119-125.

_- , satisfaction outcomes of a new treatment for somatised mental disorder taught to general practitioners. British Journal of General Practice, 49, 263-267.

Mynors-Wallis, L., Gath, D., Lloyd-Thomas, A., et al (1995) Randomised controlled trial comparing problem solving treatment with amitriptyline and placebo for major depression in primary care. British Medical Journal, 310, 441-445.

_- Day, A., et al (2000) Randomised controlled trial of problem solving treatment, antidepressant medication, and combined treatment for major depression in primary care. British Medical Journal, 320, 26-30.

Nazareth, I., King, M. \& Tai, S. S. (1996) Monitoring psychosis in general practice: a controlled trial. British Journal of Psychiatry, 169, 475-482.

Peters, S., Stanley, I., Rose, M., et al (1998) Patients' with medically unexplained symptoms: sources of patients authority and implications for demands on medical care. Social Science and Medicine, 46, 559-565.

Peveler, R., Kilkenny, L. \& Kinmonth, A.-L. (1997) Medically unexplained physical symptoms in primary care: a comparison of self-report screening questionnaires and clinical opinion. Journal of Psychosomatic Research, 42, 245-252.

Philipp, M., Kohnen, R. \& Hiller, K.-O. (1999) Hypericum extract versus imipramine or placebo in patients with moderate depression: randomised multicentre study of treatment for eight weeks. British Medical Journal, 319,1534-1539.

Rutz, W., Walinder, J., Eberhard, G., et al (1989) An education programme for depressive disorders on Gotland: background and evaluation. Acta Psychiatrica Scandinavica, 79, 19-26.

Salmon, P., Peters, S. \& Stanley, I. (1999) Patients' perceptions of medical explanations for somatisation disorders: qualitative analysis. British Medical Journal, 318, 372-376.

Scott, C., Tacchi, M. J., Jones, R., et al (1997) Acute and oneyear outcome of a randomised controlled trial of brief cognitive therapy for major depressive disorder in primary care. British Journal of Psychiatry, 171, 131-134.

Shea, M., Elkin, I., Imber, S., et al (1992) Course of depressive symptoms over follow-up: findings from the National Institute of Mental Health Treatment of Depression Collaborative Research Program. Archives of General Psychiatry, 49, 782-787.

Shepherd, M., Cooper, M., Brown, A., et al (1966) Psychiatric Illness in General Practice. Oxford: Oxford University Press.

Simon, G., Von Korff, M., Heiligenstein, J., et al (1996) Initial antidepressant choice in primary care. Journal of the American Medical Association, 275, 1897-1902.

_- - Rutter, C., et al (2000) Randomised trial of monitoring, feedback and management of care by telephone to improve treatment of depression in primary care. British Medical Journal, 320, 550-554.

Thompson, C., Kinmonth, A., Stevens, L., et al (2000) Effects of a clinical-practice guideline and practice-based education on detection and outcome of depression in primary care: Hampshire Depression Project randomised controlled trial. Lancet, 355, 185-191.

Wing, J. K., Cooper, J. E. \& Sartorius, N. (1974) Measurement and Classification of Psychiatric Symptoms: An Instruction Manual for the PSE and CATEGO Program. London: Cambridge University Press.

World Health Organization (1992) The ICD-10 Classification of Mental and Behavioural Disorders. Geneva: WHO.

\section{Multiple choice questions}

1. Research into mental health training programmes for GPs suggests that they:

a are generally unpopular with GPs

$\mathrm{b}$ can be delivered in a multidisciplinary format c reduce rates of depression in patients

d may have a positive effect on suicide prevention

e lead to greater patient satisfaction.

2. A telephone care management system for depression in primary care:

a should be administered by a pharmacologist

b should involve rapid feedback to GPs

c involves weekly contact with patients with depression

$\mathrm{d}$ is more expensive than care as usual

e can improve depression outcomes.

3. Problem-solving treatment:

a involves a long, complex training programme

b emphasises the doctor's ability to solve problems

c is unpopular with patients

$\mathrm{d}$ is less effective than antidepressants in treating depression

e can be effectively delivered by nurses.

4. Structured case management for patients with severe and enduring mental illness:

a can increase the involvement of GPs in psychiatric care

b is carried out more diligently by GPs than by nurses

c is hampered by difficulty in organising routine follow-ups

d leads to decreased consultation rates

e shows consistent benefits for patients.

5. Patients with unexplained physical symptoms:

a are identified by GPs in about one-fifth of consultations

$\mathrm{b}$ are usually depressed

c have a false understanding of their own problems

d are often involved in dysfunctional doctorpatient interactions

e usually resent attempts to link symptoms with psychosocial problems

$\begin{array}{llllllllllll}\text { MCQ answers } & & & & & & \\ \text { 1 } & & \text { 2 } & & & & & 4 & & & \\ \text { a } & \text { F } & \text { a } & \text { F } & & \text { a } & \text { F } & \text { a } & \text { T } & \text { a } & \text { T } \\ \text { b } & \text { T } & \text { b } & \text { T } & \text { b } & \text { F } & \text { b } & \text { F } & \text { b } & \text { F } \\ \text { c } & \text { F } & \text { c } & \text { F } & & \text { c } & \text { F } & & \text { c } & \text { T } & \text { c } & \text { F } \\ \text { d } & \text { T } & \text { d } & \text { T } & \text { d } & \text { F } & \text { d } & \text { F } & \text { d } & \text { T } \\ \text { e } & \text { F } & \text { e } & \text { T } & \text { e } & \text { T } & \text { e } & \text { F } & \text { e } & \text { F }\end{array}$

